\title{
A note on leveraging synergy in multiple meteorological datasets with deep learning for rainfall-runoff modeling
}

\author{
Frederik Kratzert $^{1}$, Daniel Klotz ${ }^{1}$, Sepp Hochreiter ${ }^{1}$, and Grey S. Nearing ${ }^{2,3}$ \\ ${ }^{1}$ LIT AI Lab \& Institute for Machine Learning, Johannes Kepler University Linz, Austria \\ ${ }^{2}$ Upstream Tech, Natel Energy Inc., Alameda, CA USA \\ ${ }^{3}$ Department of Geological Sciences, University of Alabama, Tuscaloosa, AL United States \\ Correspondence: Frederik Kratzert (kratzert@ml.jku.at), Grey S. Nearing (grey@upstream.tech)
}

\section{Preprint of an unreviewed manuscript}

\begin{abstract}
A deep learning rainfall-runoff model can take multiple meteorological forcing products as inputs and learn to combine them in spatially and temporally dynamic ways. This is demonstrated using Long Short Term Memory networks (LSTMs) trained over basins in the continental US using the CAMELS data set. Using multiple precipitation products (NLDAS,

5 Maurer, DayMet) in a single LSTM significantly improved simulation accuracy relative to using only individual precipitation products. A sensitivity analysis showed that the LSTM learned to utilize different precipitation products in different ways in different basins and for simulating different parts of the hydrograph in individual basins.
\end{abstract}

\section{Introduction}

There are many different meteorological products that a hydrologist might choose as forcing data, and no data product is perfect.

While temperature estimates between different forcing products are frequently similar, precipitation estimates are often subject to large disagreements (e.g., Behnke et al., 2016; Timmermans et al., 2019). The appropriate choice of the input forcing data is an important step for every modelling task. To our knowledge it is so far not possible to dissect, which methodological choices lead to which disagreements in the data products (e.g., Beck et al., 2017; Newman et al., 2019); nor is it straightforward to estimate how these differences translate to model behavior (e.g., Yilmaz et al., 2005; Henn et al., 2018; Parkes et al., 2019). Thus, the choice of the "right" product, for a given modelling exercise, requires careful consideration.

Generally speaking, the most accurate precipitation data comes from in situ gauges, which are point-based measurements of complex spatial processes (although in certain cases, especially related to snow, modeled products might be better - e.g., Lundquist et al., 2019). Today's large-scale hydrological models, however, require data fields (usually gridded), which are model-based products, resulting from a combination of spatial interpolation and/or satellite retrieval algorithms. Each product is based on different sets of assumptions that each potentially introduce different types of error and information loss. As an example, Behnke et al. (2016) showed that no existing gridded meteorological product is uniformly better than all others over the continental United States (CONUS). 
In this context, we would like to point out that - depending of the goal of the modelling exercise - data-driven models can have an inherent advantage compared to traditional hydrological modeling techniques: A single data-driven model can use multiple forcing products directly. The models can learn to exploit potential synergies in different (imperfect) precipitation data sets (or any other type of model input). In particular, deep learning models as used by Kratzert et al. (2019b, a) can take any number of different precipitation and other meteorological inputs at every timestep. Because the different input data sets are used simultaneously in a single nonparametric model, this has the potential to produce more accurate simulations by combining those inputs in spatiotemporally dynamic ways. The goal of this contribution is to test the strength of this hypothesis by assessing the model's ability to learn complex and spatiotemporally variable interactions between different precipitation products.

\section{Methods}

\subsection{Data}

This study uses the Catchment Attributes and Meteorological dataset for Large Sample Studies (CAMELS; Newman et al., 2014; Addor et al., 2017b). CAMELS contains basin-averaged daily meteorological forcing inputs derived from three different gridded data products for 671 basins across CONUS. The three forcing products are (i) DayMet (Thornton et al., 1997), (ii) Maurer (Maurer et al., 2002), and (iii) NLDAS (Xia et al., 2012), the former has $1 \mathrm{~km}$ x $1 \mathrm{~km}$ spatial resolution and the latter two have one-eighth degree spatial resolution. Although CAMELS includes 671 basins, to facilitate a direct comparison of results with previous studies we used only the subset of 531 basins that were originally chosen for model benchmarking by Newman et al. (2017), who removed all basins with area greater than $2000 \mathrm{~km}^{2}$, and also all basins where there was a discrepancy of more than $10 \%$ between different methods of calculating basin area. These 531 basins were used for all experiments in this study except benchmarking against traditional hydrology models (see Sect. 2.4.1), because the benchmark models are only available at 447 of the 531 basins.

Behnke et al. (2016) conducted a detailed analysis of eight different precipitation and surface temperature (daily $\mathrm{max} / \mathrm{min}$ ) data products, including the three used by CAMELS. Those authors compared gridded precipitation and temperature values to station data using roughly 4000 weather stations across CONUS. Their findings were that "no data set was 'best' everywhere and for all variables we analyzed" and "two products stood out in their overall tendency to be closest to (Maurer) and farthest from (NLDAS2) observed measurements." Furthermore, they did not find a "clear relationship between the resolution of gridded products and their agreement with observations, either for average conditions ... or extremes" and noted that the "high-resolution DayMet ... data sets had the largest nationwide mean biases in precipitation."

Figure 1 gives an example of disagreement between precipitation products in CAMELS that we hope to capitalize on by training a model with multiple forcing inputs. This figure shows the noisy relationship between the three precipitation products in a randomly-selected basin (USGS ID: 07359610). Deep Learning approaches can learn to mitigate the type of noise shown in the scatter plot in the right-hand panel of Fig. 1 and use the inherent information by using multiple forcing products simultaneously in a single model. 

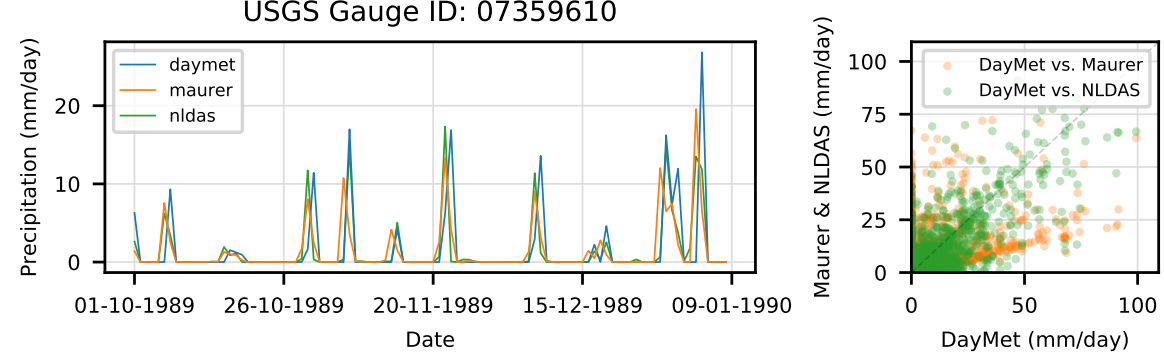

Figure 1. Illustration of the relationship between three CAMELS precipitation products at a randomly-selected basin. The left-hand subplots show the first 100 days of precipitation data from all three products during the test period, and the right-hand subplot shows scatter between the three products over the full test period. The scatter shown in the right-hand subplot is the data uncertainty that we would like to mitigate by using multiple forcings simultaneously in a deep learning rainfall-runoff model. In this particular basin, there appears to be a 1-day shift between DayMet and Maurer, which is common in the CAMELS data set (this shift is apparent in 325 of the 531 basins; see Figure 2)

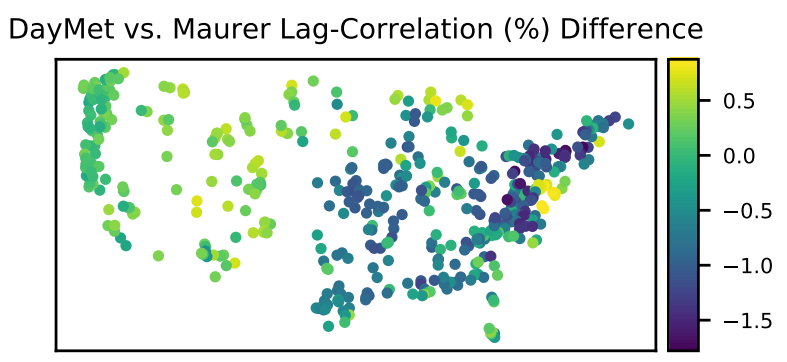

Figure 2. Spatial distributions of lagged vs. non-lagged correlations between DayMet and Maurer test-period precipitation. Positive values indicate that the 1-day lagged correlation is higher.

The left-hand subplot of Fig. 1 shows a time-shift between DayMet and Maurer precipitation in the same basin. This type of shift is common. Behnke et al. (2016), for example, reported that "[b]ecause gridded products differ in how they define a calendar day (e.g., local time relative to Coordinated Universal Time), appropriate lag correlations were applied through cross- correlation analysis to account for the several- hour offset in daily station data." We performed a lag-correlation analysis on the precipitation products in CAMELS and found a higher correlation between DayMet and Mauer when Mauer was lagged by one day in 325 (of 531) basins. Figure 2 shows the percent difference between lagged vs. non-lagged correlations between DayMet and Maurer.

Each of the forcing products in CAMELS includes daily precipitation $(\mathrm{mm} / \mathrm{d})$ and maximum and minimum daily temperature $\left({ }^{\circ} \mathrm{C}\right)$, vapor pressure $(\mathrm{Pa})$, and surface radiation $\left(\mathrm{W} / \mathrm{m}^{2}\right)$. The original CAMELS data set hosted by the US National Center for Atmospheric Research (Newman et al., 2014) only contains daily mean temperatures for Maurer and NLDAS. CAMELSrelevant Maurer and NLDAS products with daily minimum and maximum temperatures are available from our HydroShare 
DOI (see data availability section). In addition to the three daily forcing data sets from CAMELS, we used the same 27 catchment attributes as Kratzert et al. (2019a, b), which consist of topological, climatic, vegetation, and soil descriptors (Addor et al., 2017a). Prior to training any model, all input variables were normalized independently by subtracting the CONUS-wide mean and dividing by the CONUS-wide standard deviation.

\subsection{Models}

Long Short-Term Memory networks (LSTMs) are a type of recurrent neural network (Hochreiter, 1991; Hochreiter and Schmidhuber, 1997b; Gers et al., 1999). LSTMs are a type of state-space model that function through a set of input-stateoutput relationships. Gates, which are activated linear functions, control information flows from inputs and previous states to current state values (called an input gate), from current states to outputs (called an output gate), and also control the timescale of each element of the state vector (called a forget gate). States (usually called cell states) accumulate and store information over time, much like the states of a dynamical system. Technical details of the LSTM model architecture have been described in several previous publications in hydrology journals, and we refer the reader to Kratzert et al. (2018) for a detailed explanation geared towards hydrologists.

\subsection{Experimental Design}

To conduct our analyses we trained an LSTM model using each of the three forcing products together, separate LSTM models for each pairwise combination of forcing products (DayMet \& Maurer, DayMet \& NLDAS, and Maurer \& NLDAS), and separate LSTMs for all three forcing products individually.

For each of these seven input configurations, we trained an ensemble of $n=10$ different LSTMs with different randomly initialized weights. We report the statistics from averaging the simulated hydrographs from each of these 10-member ensembles (single model results are provided in the Appendix). Ensembles are used to account for randomness inherent in the training procedure. The importance of using ensembles for this purpose was demonstrated by Kratzert et al. (2019b).

We used the same time periods for model training and testing as by Kratzert et al. (2019b) - this allows us to directly compare results of this study with the full set of benchmark hydrology models used by that previous study. The training period was from 1 October 1999 to 30 September 2008 (9 years of training data for each catchment) and the test period was 1 October 1989 to 30 September 1999 (10 years of test data for each catchment).

Similar to previous studies (Kratzert et al., 2019b, a), we used LSTMs with 256 memory cells and a dropout rate of $0.4(40 \%)$ in the fully connected layer that derives network predictions (streamflow) from LSTM output. All models were trained with a mini-batch size of 256 for 30 epochs using the Adam optimizer (Kingma and Ba, 2014) with an initial learning rate of 1e-3, reduced to 5e-4 after 20 epochs and further reduced to 1e-4 after 25 epochs. All inputs were standardized to have zero mean and unit variance over all 531 catchments collectively. During model evaluation, negative predictions in the original value space were clipped to zero, i.e. no negative discharges. The loss function was the basin-averaged Nash-Sutcliffe Efficiency (NSE), see Kratzert et al. (2019b). 


\subsection{Analysis}

100

We examined the experiments described above with three types of analysis. The goal is to provide different illustrations of how the LSTM leveraged multiple forcing products.

- Analysis 1 - Feature Ablation: An ablation study removes parts of the network to gain a better understanding of the model. We adopted this procedure by removing the different meteorological forcing products in a step-wise fashion and comparing the individual results by using multiple performance metrics and signatures (see Table 1). To provide context we also compare them against a family of conceptual and process-based hydrological models.

- Analysis 2 - Precipitation Uncertainty: We used triple collocation to estimate spatially-varying error characteristics of the three precipitation forcing products, and assessed relationships between these error statistics with the performance of both single- and multiple-forcing LSTMs. These experiments help us understand where we can expect value from using multiple forcing products in a single model.

- Analysis 3 - Sensitivity \& Contribution: We performed an input attribution analysis of the trained LSTM models to quantify how the LSTM learned to leverage different forcing products in spatiotemporally dynamic ways.

\subsubsection{Analysis 1: Feature Ablation}

All LSTM ensembles were trained using a squared-error loss function (the basin-averaged NSE), however we are interested to know how the models simulate different aspects of the hydrograph. As such, we report a collection of hydrologically-relevant performance metrics outlined in Table 1. These statistics include the standard time-average performance metrics (NSE, KGE), as well as comparisons between observed and simulated hydrologic signatures. The hydrologic signatures we report are the same ones used by Addor et al. (2018). For each signature, we compute the Pearson correlation between the signature derived from the observed discharge and derived from the simulated discharge of all basins.

To provide a baseline for comparison, LSTM ensembles were benchmarked against the same family of hydrological models used for benchmarking by Kratzert et al. (2019b). These models are: (i) SAC-SMA (Burnash et al., 1973; Burnash, 1995) coupled with the Snow-17 snow routine (Anderson, 1973), hereafter referred to as SAC-SMA, (ii) VIC (Liang et al., 1994), (iii) FUSE (Clark et al., 2008; Henn et al., 2008) (three different model structures, 900, 902, 904), (iv) HBV (Seibert and Vis, 2012), and (v) mHM (Samaniego et al., 2010; Kumar et al., 2013). Some of these models were calibrated to individual basins and others were regionally calibrated. All of the benchmarks used Maurer forcings and all were calibrated and validated on the same time periods used in this study. In order to avoid any potential or implicit bias, we did not run any of our own benchmark models - all models were solicited originally by Kratzert et al. (2019b) from different groups with experience running each individual model. The whole family of benchmark model runs is only available in 447 of the 531 CAMELS catchments, chosen by Newman et al. (2017). Thus, while we use the set of 531 catchments for all other parts of this study, we only considered 447 catchments for benchmarking against traditional hydrology models. 
Table 1. Description of the performance metrics (top part) and signatures (bottom part) considered in this study. For each signature, we derived a metric by computing the Pearson correlation between the signature of the observed flow and the signature of the simulated flow over all basins. Description of the signatures taken from Addor et al. (2018)

\begin{tabular}{|c|c|c|}
\hline Metric/Signature & Description & Reference \\
\hline NSE & Nash-Sutcliff efficiency & Eq. 3 in Nash and Sutcliffe (1970) \\
\hline KGE & Kling-Gupta efficiency & Eq. 9 in Gupta et al. (2009) \\
\hline Pearson rt & Pearson correlation between observed and simulated flow & \\
\hline$\alpha$-NSE & Ratio of standard deviations of observed and simulated flow & From Eq. 4 in Gupta et al. (2009) \\
\hline$\beta$-NSE & Ratio of the means of observed and simulated flow & From Eq. 10 in Gupta et al. (2009) \\
\hline FHV & Top $2 \%$ peak flow bias & Eq. A3 in Yilmaz et al. (2008) \\
\hline FLV & Bottom $30 \%$ low flow bias & Eq. A4 in Yilmaz et al. (2008) \\
\hline FMS & Bias of the slope of the flow duration curve between the $20 \%$ and $80 \%$ percentile & Eq. A2 Yilmaz et al. (2008) \\
\hline Peak-Timing & Mean peak time lag (in days) between observed and simulated peaks & See Appendix C \\
\hline Baseflow index & Ratio of mean daily baseflow to mean daily discharge & Ladson et al. (2013) \\
\hline HFD mean & $\begin{array}{l}\text { Mean half-flow date (date on which the cumulative discharge since October first reaches } \\
\text { half of the annual discharge) }\end{array}$ & Court (1962) \\
\hline High flow dur. & $\begin{array}{l}\text { Average duration of high-flow events (number of consecutive days }>9 \text { times the } \\
\text { median daily flow) }\end{array}$ & Clausen and Biggs (2000), Table 2 in Westerberg and McMillan (2015) \\
\hline High flow freq. & Frequency of high-flow days ( $>9$ times the median daily flow) & Clausen and Biggs (2000), Table 2 in Westerberg and McMillan (2015) \\
\hline Low flow dur. & $\begin{array}{l}\text { Average duration of low-flow events (number of consecutive days }<0.2 \text { times the } \\
\text { mean daily flow) }\end{array}$ & Olden and Poff (2003), Table 2 in Westerberg and McMillan (2015) \\
\hline Low flow freq. & Frequency of low-flow days $(<0.2$ times the mean daily flow $)$ & Olden and Poff (2003), Table 2 in Westerberg and McMillan (2015) \\
\hline Q5 & $5 \%$ Flow quantile (low flow) & \\
\hline Q95 & 95\% Flow quantile (high flow) & \\
\hline Q mean & Mean daily discharge & \\
\hline Runoff ratio & Runoff ratio (ratio of mean daily discharge to mean daily precipitation, using DayMet precipitation) & Eq. 2 in Sawicz et al. (2011) \\
\hline Slope FDC & Slope of the flow duration curve (between the log-transformed 33rd and 66th streamflow percentiles & Eq. 3 in Sawicz et al. (2011) \\
\hline Stream elasticity & $\begin{array}{l}\text { Streamflow precipitation elasticity (sensitivity of streamflow to changes in precipitation at } \\
\text { the annual time scale, using DayMet precipitation) }\end{array}$ & Eq. 7 in Sankarasubramanian et al. (2001) \\
\hline Zero flow freq. & Frequency of days with zero discharge. & \\
\hline
\end{tabular}

\subsubsection{Analysis 2: Precipitation Uncertainty}

The objective of the second analysis is to demonstrate that the multiple-forcing model learns to leverage patterns in forcing data error structures. Our approach was to relate error characteristics of the different precipitation products with model performance, and with performance improvements due to using multiple forcing products. We used triple collocation to estimate error characteristics of the different forcing products. Triple collocation is a statistical technique to estimate error variances of three or more noisy measurement sources without knowing the true values of the measured quantities (Stoffelen, 1998; Scipal et al., 2010). Its major assumptions are that the error models are linear and independent between sources; in particular, that all (three or more) measurement sources are each a combination of a scaled value of the true variable plus some additive random noise:

$$
M_{i, t}=\alpha_{i} T_{t}+\varepsilon_{i, t},
$$


where $M_{*}$ are measurement values (i.e. here the modeled precipitation values), subscript $i$ represents the source (DayMet, Maurer, NLDAS), and subscript $t$ represents the timestep in the test period (1 October 1989 to 30 September 1999); $T_{*}$ is the unobserved true value of total precipitation in a given catchment on a given day; $\varepsilon_{*}$ are i.i.d. measurement errors from any distribution.

The linearity assumption is not appropriate for precipitation data, which are typically assumed to have multiplicative error. Following Alemohammad et al. (2015), we assumed a multiplicative error model for all three precipitation source, and converted these to linear error models by working with the log-transformed precipitation data:

$$
\begin{gathered}
M_{i, t}=\alpha_{i} T_{t}^{\beta_{i}}+e^{\varepsilon_{i, t}} \\
\ln \left(M_{i, t}\right)=\alpha_{i}+\beta_{i} T_{t}+\varepsilon_{i, t} .
\end{gathered}
$$

Standard triple collocation is then applied, so that estimates of the error variances for each source are:

$\sigma_{i}=C_{i, i}-\frac{C_{i, j} C_{i, k}}{C_{j, k}}$,

for all $i, j, k$, where $C_{i, j}$ is the covariance between the time series of source $i$ and source $j ; \sigma_{i}$ is the variance of the distribution that each i.i.d. $\varepsilon_{i, t}$ is drawn from.

Additionally, extended triple collocation (McColl et al., 2014) allows us to derive the correlation coefficients between measurement sources and truth as:

$\rho_{i}=\frac{C_{i, j} C_{i, k}}{C_{i, i} C_{j, k}}$.

This triple collocation analysis was applied separately in each of the 531 CAMELS catchments to obtain basin-specific estimates of the error variances, $\sigma_{i}$, and truth-correlations, $\rho_{i}$, for each of the three precipitation products. Albeit the assumption that the forcing products have independent error structures (i.e. $\varepsilon_{i, t} \Perp \varepsilon_{j, t}$ ) is not met in our case we expect the results to be robust enough for the purpose at hand.

\subsubsection{Analysis 3: Sensitivity \& Contribution}

All neural networks (like LSTMs) are differentiable almost everywhere by design. Therefore, a gradient-based contribution analysis seems natural. However, as discussed by Sundararajan et al. (2017), the naive solution of using local gradients is not a reliable measures of sensitivity, since gradients might be flat even if the model response is heavily influenced by a particular input data source (which is not by necessity a bad property, see for example Hochreiter and Schmidhuber, 1997a). This is especially true in neural networks, where activation functions often include step-changes over portions of the input space - e.g., the sigmoid and hyperbolic tangent activation functions used by LSTMs have close-to-zero gradients at both extremes (see also: Shrikumar et al., 2016; Sundararajan et al., 2017).

Sundararajan et al. (2017) proposed a method of input attribution for neural networks which accounts for this described lack of local sensitivity: integrated gradients. Integrated gradients are a path integral of the gradients from some baseline input $x^{\prime}$, 
to the actual value of the input, $x$ :

$\operatorname{IntegratedGrads}_{i}^{\operatorname{approx}}(\boldsymbol{x}):=\left.\frac{\mathbf{x}_{i}-\mathbf{x}_{i}^{\prime}}{m} \sum_{k=1}^{m} \frac{\partial F(\tilde{\mathbf{x}})}{\partial \tilde{\mathbf{x}}_{i}}\right|_{\tilde{\mathbf{x}}=\mathbf{x}^{\prime}+\frac{k}{m}\left(\mathbf{x}-\mathbf{x}^{\prime}\right)}$

We used a value of zero precipitation everywhere as the baseline for calculating integrated gradients with respect to the three different precipitation forcings (DayMet, Maurer, NLDAS). We calculated the integrated gradients of each daily streamflow estimate in each CAMELS basin during the 10-year test period with respect to precipitation inputs from the past 365 days (the look-back period of the LSTM). That is, on day $t=T$, we calculated $1095=3 * 365$ integrated gradient values related to the three precipitation products. The relative integrated gradient values quantify how the LSTM combines precipitation products over time, over space, and also as a function of lag or lead-time into the current streamflow prediction. In theory, one has to take into account the effect of "explaining away", when analysing the decision process in models (Pearl, 1988; Wellman and Henrion, 1993). However, we assume that if evaluated over hundreds of basins and thousands of time steps, this effect is largely averaged out and therefore the analysis provides an indication of the actual information used by the model.

\section{Results \& Discussion}

\subsection{Results: Analysis 1 - Feature Ablation}

The feature ablation analysis compared NSE values over 10-year test periods from the CAMELS basins for the seven distinct input combinations. As shown in Fig. 3, the three-forcing model had a median NSE value of 0.82 for the 447 basins, which were available for all benchmarking models. The three-forcing model outperformed all two-forcing models. Similarly, all twoforcing models outperformed all single-forcing models (all improvements were statistically significant at $\alpha=0.001$, using the Wilcoxon test). The best single-forcing LSTM had a median NSE of 0.76. This indicates that the LSTM was able to leverage unique information in the precipitation signals (this is not an unusual finding in the context of machine learning, see for example: Sutton, 2019). We also note that the single-forcing LSTM with Maurer inputs outperformed the single-forcing NLDAS model, which agrees with the results of Behnke et al. (2016) who showed that Maurer precipitation was generally more accurate than NLDAS precipitation.

To put these results into context, Fig. 4 compares all LSTMs against the benchmark hydrology models in the 447 basins where simulations of all benchmark models were available. All LSTM models were better than all benchmark models through the entire CDF curve. As a point of reference, the difference in the median NSE between the best-performing single-forcing LSTM (DayMet) and the best-performing traditional hydrology model (HBV) was 0.09, while using all three CAMELS forcings increased that improvement over traditional models by another $0.055(61 \%)$. The total improvement in the median NSE of the multi-forcing LSTM over the best-performing hydrology model was 0.143 (21\%).

Table 2 and Table 3 give the benchmarking results from all metrics and signatures in Table 1. The three-forcing LSTM out-performs all benchmark models against all metrics except $\beta$-NSE decomposition, the bias of the slope of the flow duration curve (FMS) and the bias of the low flows (FLV). The three-forcing LSTM also out-performs all benchmark models against all 


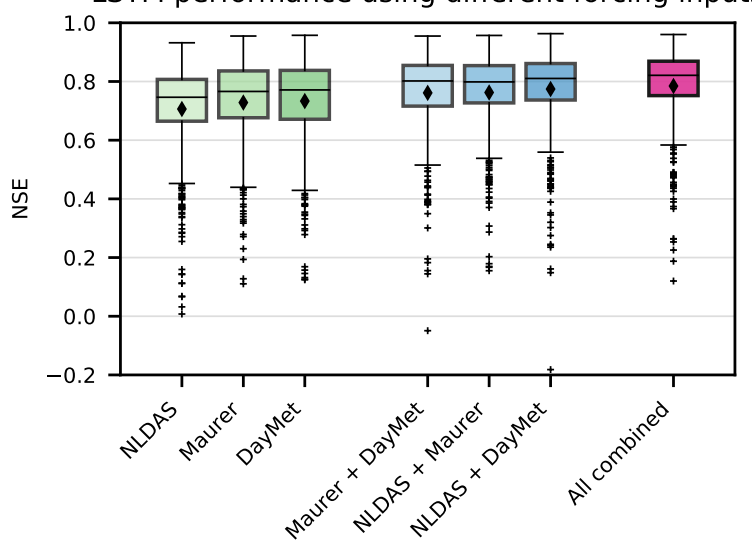

Figure 3. Test-period comparison between single-forcing and multiple-forcing LSTM ensembles $(n=10)$ over 447 CAMELS basins. All differences are statistically significant $(\alpha=0.001)$, with the exceptions of "DayMet" vs. "Maurer" $(p \approx 0.08)$ and "NLDAS + Maurer" vs. "Maurer + DayMet" $(p \approx 0.4)$

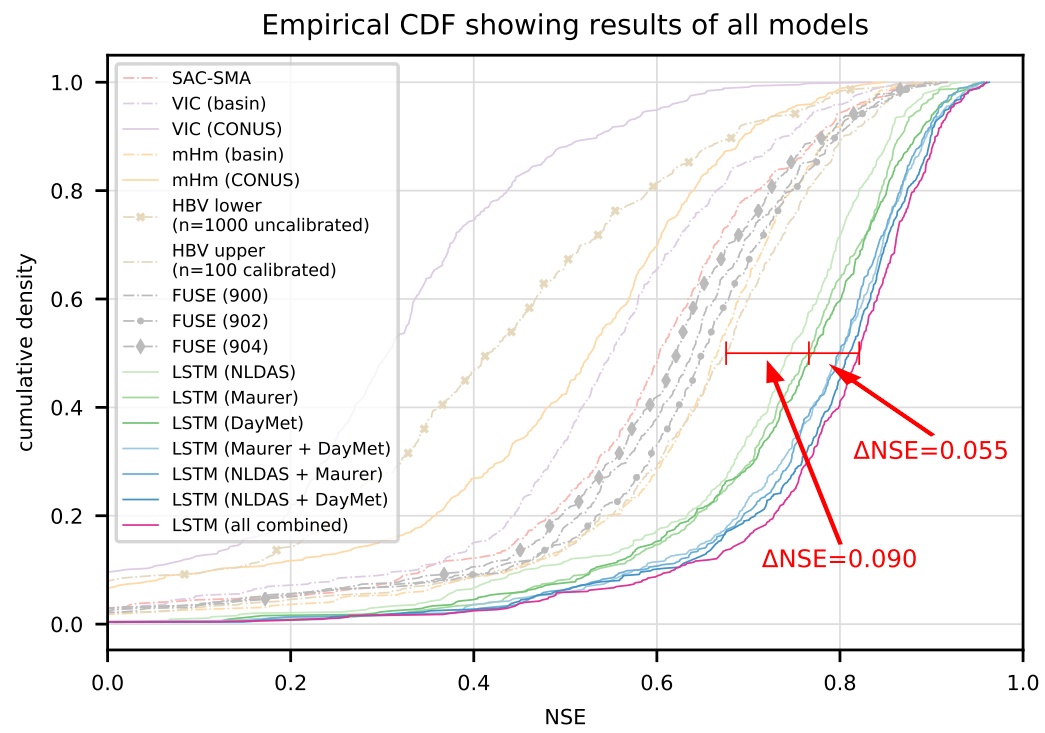

Figure 4. Empirical cumulative density function of the NSE performance over the 447 commonly modelled basins of all LSTMs and benchmark models. The red indicator lines mark the median NSE difference between the best hydrological benchmark model (ensemble of 100 calibrated HBV models) and the LSTM of our previous publication (Kratzert et al., 2019b), as well as the current best, if trained with all forcing products combined. 

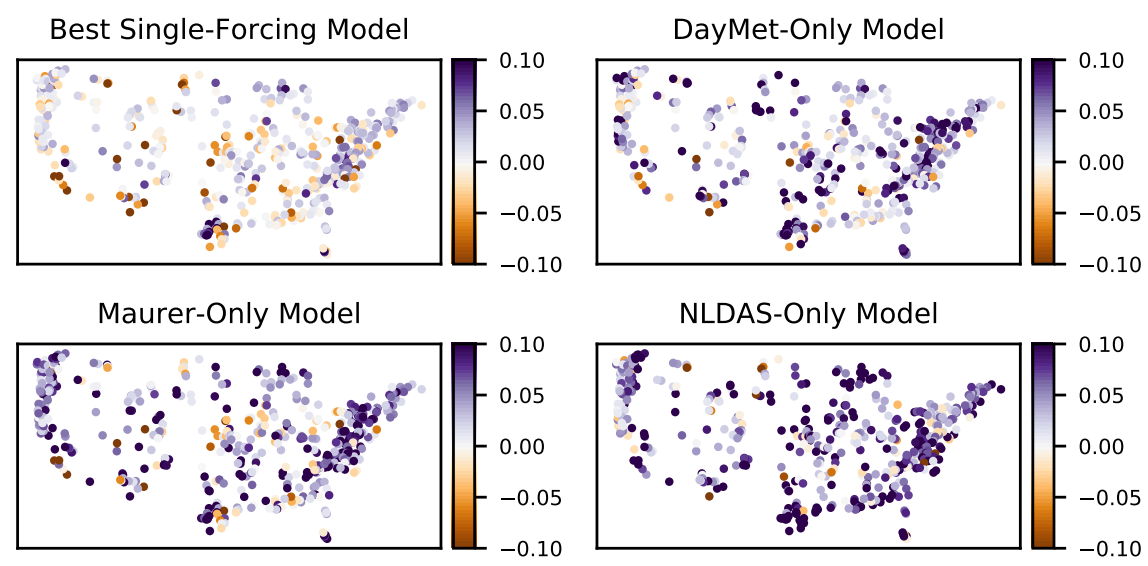

Figure 5. Spatial distribution of the NSE differences between the three-forcing LSTM relative to the best single-forcing model in each basin (top-left subplot), and relative to each single-forcing model (other three subplots). The three-forcing LSTM was better than the best singleforcing model in 351 of 531 basins (66\%) and was better than each single-forcing model in: 443 (83\%; DayMet), 456 (86\%; Maurer), and $472(89 \%$; NLDAS) basins.

hydrologic signatures except the ones related to low-flows (frequency of zero flows and frequency and duration of flows below $20 \%$ of basin-average). We therefore note that the LSTM approach - while generally providing the best available model - still has approximation difficulties towards the extreme lower-end of the runoff distribution.

Looking at the spatial distribution of the performance differences in all basins used for model training (i.e., 531 basins instead of the 447 basins used for the benchmarking described above), it is evident that the three-forcing LSTM outperformed the single forcing models almost everywhere (Fig. 5). Individual exceptions where "less is more" do however exist (e.g. Southern California). Concretely, the three-forcing model was better than the best single forcing model in 66\% of the basins (351 of 531) and had a higher NSE than the individual single-forcing LSTMs in over $80 \%$ of the basins

\subsection{Results: Analysis 2 - Precipitation Uncertainty}

DayMet typically produces lower NSE values in basins where triple collocation reported that the DayMet precipitation error variances are high. This is what we would expect: low skill in basins with high precipitation error; however we did not see similar patterns with the other two precipitation products (see Fig. 6, where the triple collocation error variances and truthcorrelation are plotted against the NSE scores of the single-source models) . In fact, the NLDAS LSTM tends to perform worse in basins with lower precipitation error (as estimated by triple collocation).

Figure 7 is an adapted version of Fig. 6 that highlights a few high-skill, high-variance NLDAS basins in blue. These basins correspond to a cluster of basins in the Rocky Mountains (Fig. 8) where NLDAS has low correlation with the other two products but still yields high-skill LSTM simulations. 


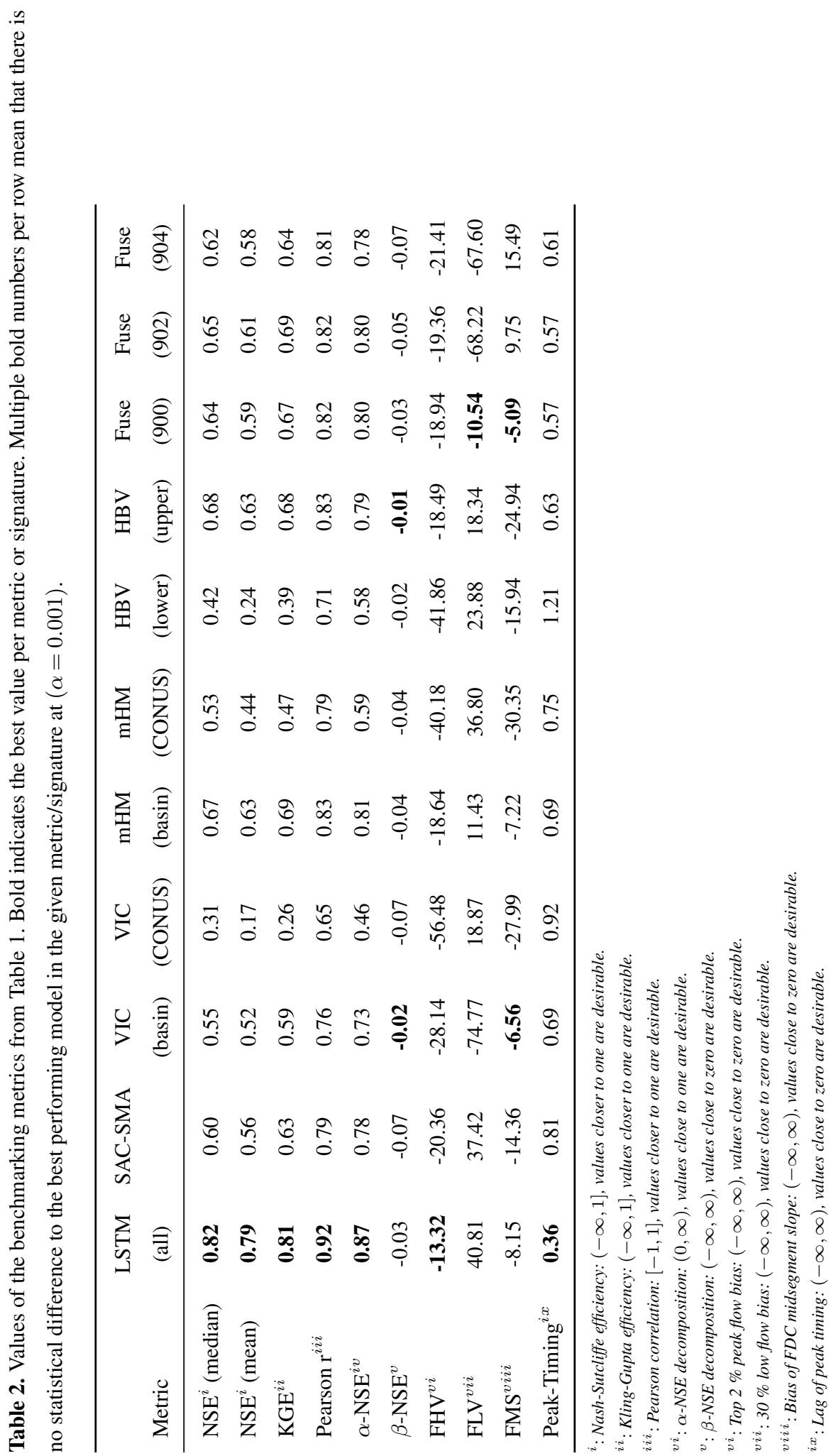




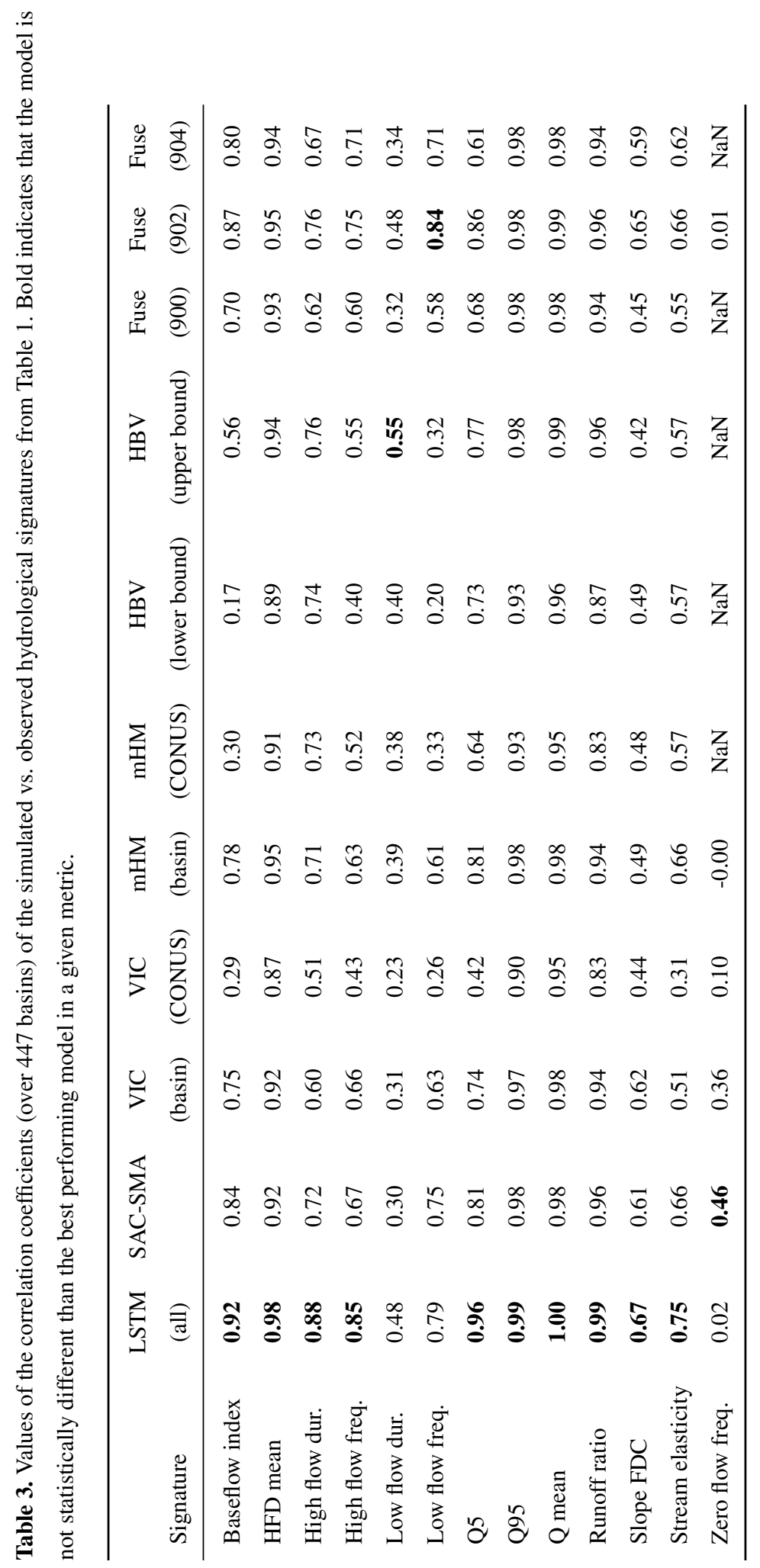




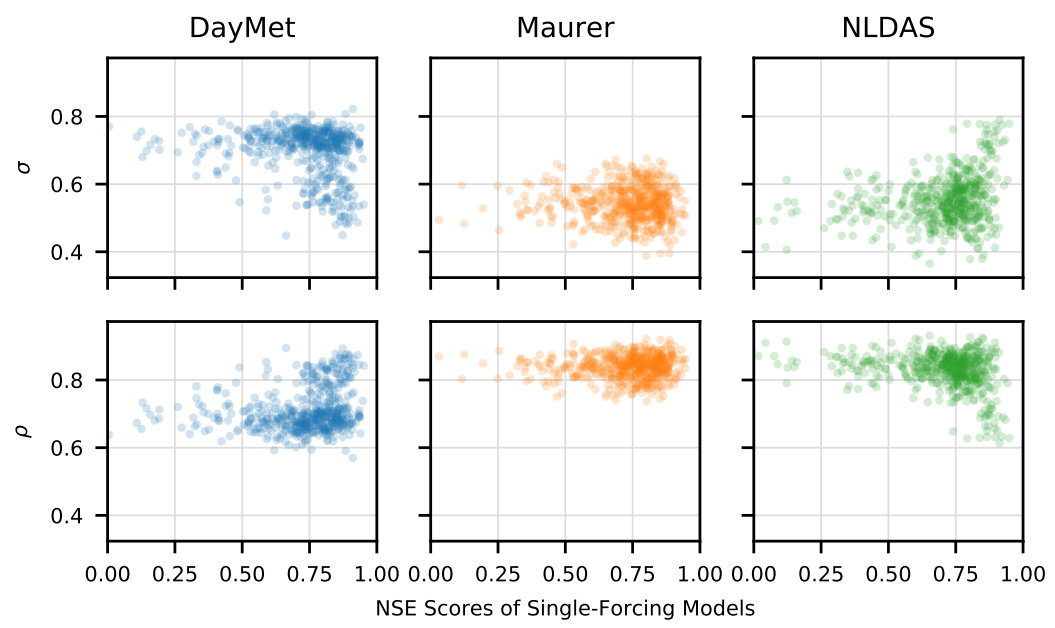

Figure 6. Triple collocation error variances and truth-correlations plotted against NSE scores of the single-forcing LSTM models. DayMet typically produces lower NSE values in basins where triple collocation reports that the precipitation error variances are high, whereas NLDAS produces lower NSE values in basins where triple collocation reports that the error variances are low. There is no apparent pattern in the Maurer data.
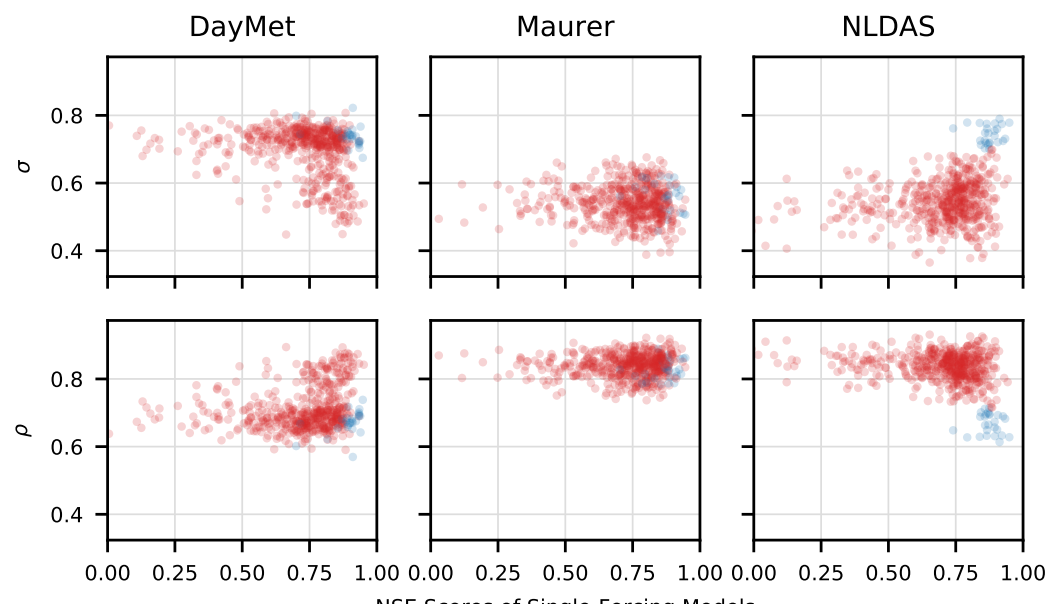

NSE Scores of Single-Forcing Models

Figure 7. As in Fig. 6 the triple collocation error variances and truth-correlations are plotted against NSE scores of the single-forcing LSTM models. The coloring shows the anomalous NLDAS basins in blue and all others in red. For these basins NLDAS has low correlation with the other two products but still yields high-skill simulations.

Triple collocation measures (dis)agreement between measurement sources, rather than error variances directly. Figure 9 plots model performance against the individual variances of the precipitation products in each basin. This figure shows that the single-forcing DayMet LSTM tends to perform better in catchments with higher total precipitation variance (not triple 
Anomalous NLDAS Basins

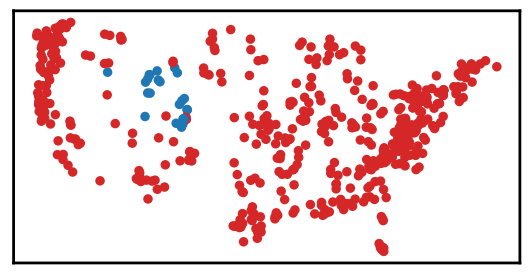

Basin Elevation [m]

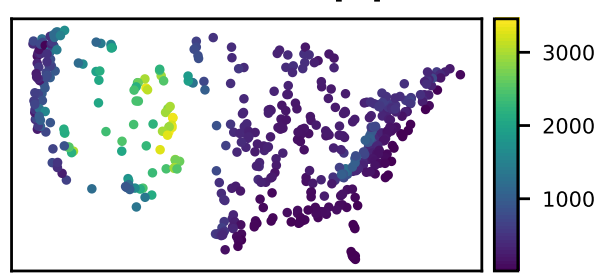

Figure 8. Spatial distribution of anomalous NLDAS basins shown in Fig. 7 (left) compared with elevation of the CAMELS basins (right).
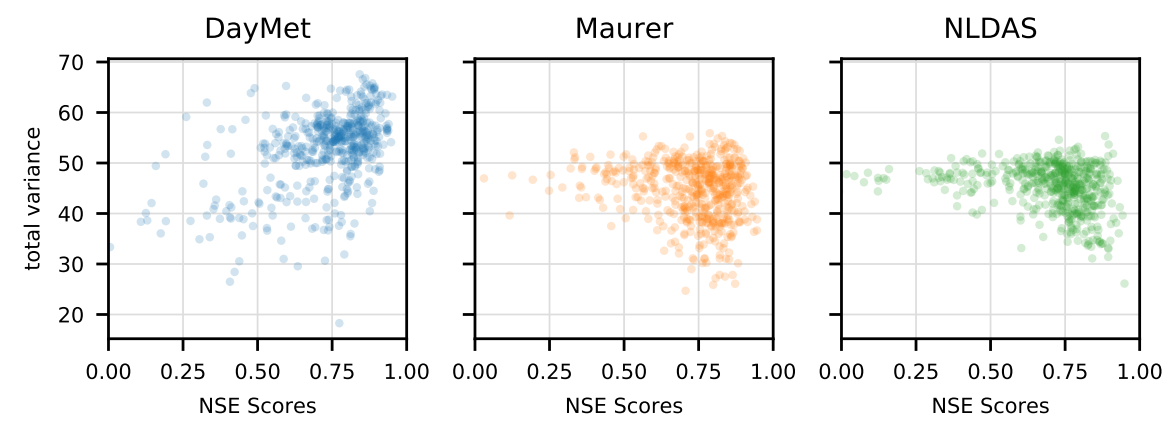

Figure 9. Performance of single-input models relative to the total variance of log-precipitation in each basin. The DayMet model tends to perform better in wetter basins (as the total DayMet variance increases), but the other two products have poor performing basins in catchments with high precipitation variance.

collocation error variance), indicating better performance in wetter catchments. This is not true for the other two models, where higher total variance is associated with a higher variance in model skill, indicating higher proportion of the variance due to measurement error.

To analyse the synergy due to using all forcings in a single LSTM we transposed the NSE improvements in each basin (due to using all three forcing products in the same LSTM) with the log-determinant of the covariance matrix of all three (standardized, log-transformed) precipitation products (Fig. 10). The log-determinant is a proxy for the joint entropy of the three (standardized, log-transformed) products, and increases when there is larger disagreement between the three data sets. Unlike in Fig. 9, the variances in Fig. 10 were calculated after removing the mean and overall variance of each log-transformed precipitation product so that the log-determinant of the covariance is not affected by the overall magnitude of precipitation in each catchment (i.e., does not increase in wetter catchments). With the exception of the anomalous NLDAS basins, Fig. 10 shows that the three-forcing model offers improvements with respect to the single-forcing models when there is larger disagreement between the three data sets. 

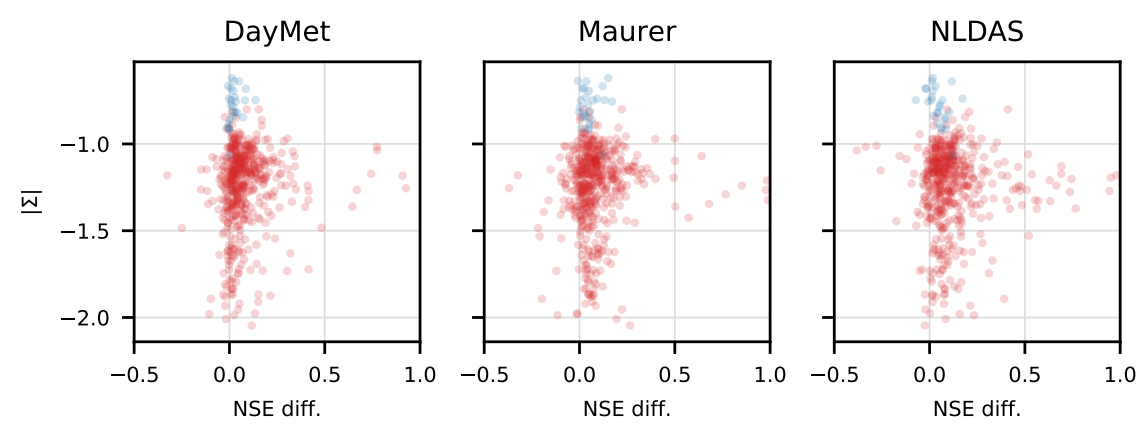

Figure 10. Fractional increase in NSE from the three-forcing model relative to the single-forcing models plotted against the log-determinant of the covariance matrix of all three (standardized, log-transformed) precipitation products. With the exception of the anomalous NLDAS basins (blue markers), the three-forcing model offers improvements with respect to the single-forcing models when there is larger disagreement between the three data sets. The three-forcing model learned to leverage synergy in these three precipitation products.

\subsection{Results: Analysis 3 - Sensitivity \& Contribution}

Figure 11 shows the time- and basin-averaged integrated gradient of the multi-forcing LSTM as a function of lead time. To reiterate from above, the integrated gradient is a measure of input attribution, or sensitivity such that inputs with higher integrated gradients have a larger influence on model outputs. Integrated gradients shown in Fig. 11 were averaged over all timesteps in the test period, and also over all basins. This figure shows the sensitivity of streamflow at time $t=T$ to each of the three precipitation inputs at times $t=T-s$ where $s$ is the lag value on the x-axis. The main takeaways from this high-level illustration of the input sensitivities are: (1) that the sensitivity of current streamflow to precipitation decays with lead time (i.e., time before present) and (2) that the multi-forcing model has learned to ignore the Maurer input at the present timestep. The reason for the latter is the time shift in the Maurer product illustrated in Fig. 2.

The multi-forcing LSTM learned to combine the different precipitation products in spatiotemporally variable ways. Fig. 11 demonstrates the overall behavior of the multi-forcing LSTM. It is, however a highly condensed aggregate of a highly non-linear system. As such a lot of specific information is lost - as is always the case when nonlinearities are aggregated.

Therefore, Fig. 12 details the overall model behavior (through the lense of integrated gradients) by basin, and up to a lead time of $s=3$ days prior to present. The model largely ignores Maurer precipitation at the current timestep in most basins - as is already apparent in Fig. 11, but the ratio of the contributions of each product varies between basin. Figure 12 shows relative contributions of each precipitation product, but the overall importance of precipitation also varies between basin.

Similarly, Fig. 13 shows the spatial extend of the most sensitive contribution over all time steps (left-subplot) and the the overall sensitivity to all three products combined (right-subplot), which is highly correlated with the total (or average) precipitation in the basin.. That is, they display the sum of the integrated gradients over time, lag, and product. From the rightsubplot it becomes evident that the precipitation has a larger contribution to the sensitivity of streamflow predictions in wetter basins. Figure 13 also shows the product with the highest overall contribution in each basin. 


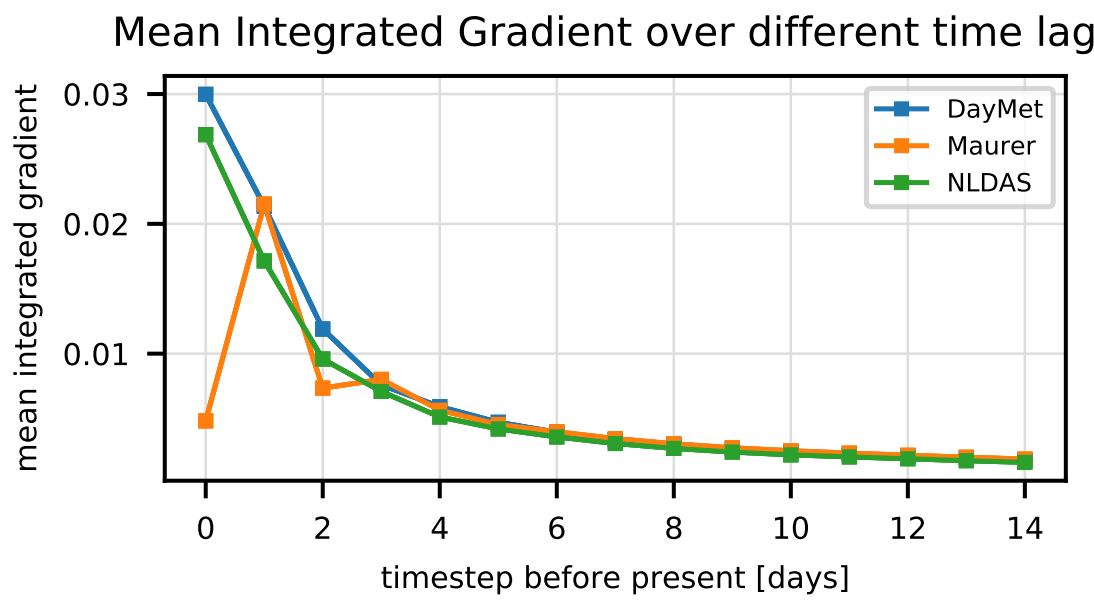

Figure 11. Time- and basin-averaged integrated gradients as a function of lag time (days before current streamflow prediction) of the three precipitation products. Because of the time shift shown in Fig. 2, the model has learned to ignore the Maurer input at the current timestep.

It is possible to break the spatial relationship down even further. Concretely, we did examine at the spatial distribution of the highest-ranked product as a function of the lag time for rising and falling limits. We can then see that the multi-forcing LSTM learns to combine the different products in very nuanced ways, distinguishing between different memory timescales in different basins for different hydrological conditions (Fig. 14).

\section{Conclusions}

The purpose of this paper is to show how LSTMs can leverage different precipitation products in spatiotemporally dynamic ways to improve streamflow simulations. The experiments show that there exist systematic and location- and time- specific differences between different precipitation products that can be learned and leveraged by deep learning. As might be expected, the LSTMs tested here tended to improve hydrological simulations more when there were larger disagreement between different precipitation estimates in a given basin.

It is worth comparing these findings with classical conceptual and process-based hydrological models that treat precipitation estimate as an unique input. Current best-practice for using multiple precipitation products is to run an ensemble of hydrological models, such that each forcing data set is treated independently. Deep learning models not only have the ability to use a larger number and variety of inputs than classical hydrology models. As a matter of fact, deep learning models do not need inputs that represent any given hydrological variable or process, and therefore have the potential to use less highly processed input data. Future work might focus on building runoff models that take as inputs the raw measurements that were used to create standard precipitation data products. 

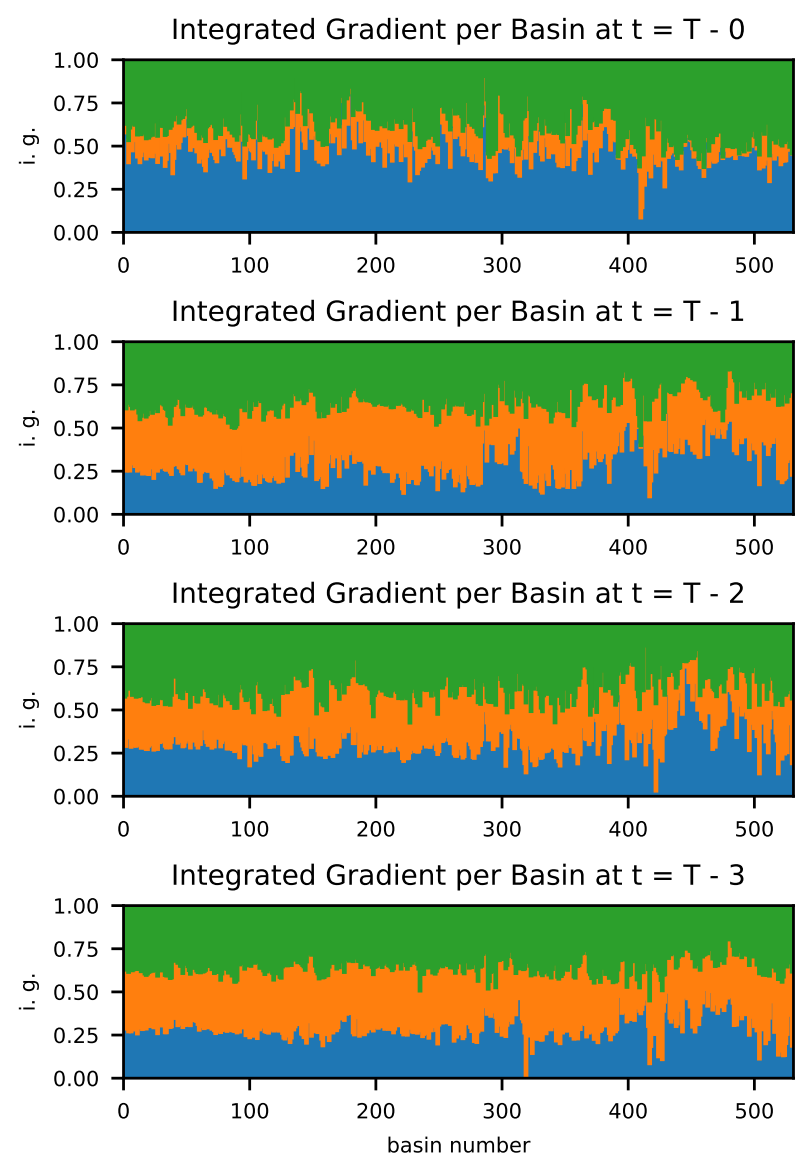

Figure 12. Expansion of Fig. 11 by individual basins, truncated at a lag of $s=3$. The multi-forcing LSTM combined the precipitation products in different ways in different basins. DayMet is generally more important in high-number basins, located in the Pacific Northwest

Deep learning provides possibilities not only for improving the quality of regional (Kratzert et al., 2019b) and even ungauged (Kratzert et al., 2019a) simulations, but also potentially for replacing large portions of ensemble-based strategies for uncertainty quantification (e.g. Clark et al., 2016) with multi-input models. There are many ways to deal with the uncertainty in traditional hydrological modeling workflows. Arguably, the most common approach is to use ensembles (e.g., Clark et al., 2016). Ensembles can be either opportunistic - i.e., from a set of pre-existing models or data products - or constructed - i.e., sampled from a probability distribution - (Clark et al., 2016), but in either case the idea is to use variability to represent lack of perfect information. Multi-input deep learning has the potential to provide a fundamentally alternative method for assessing this kind of uncertainty. Future work should additionally focus on producing predictive probabilities with multi-input deep learning models. 

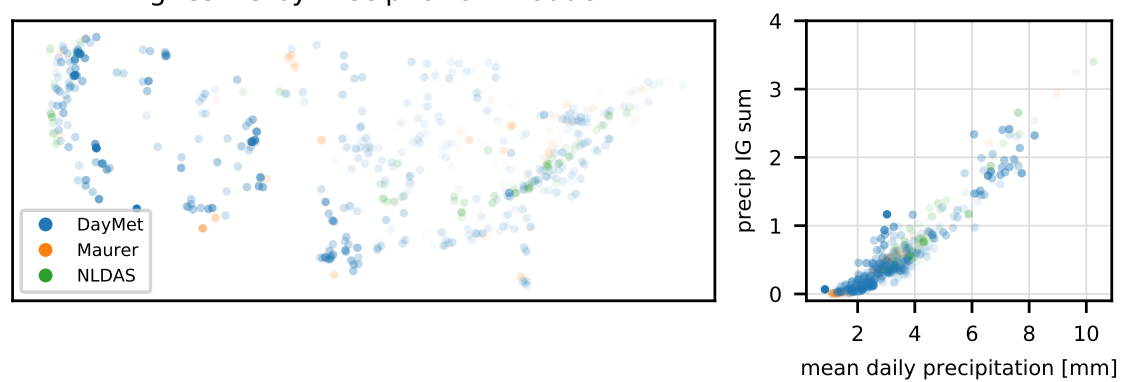

Figure 13. The forcing product with highest overall contribution (sensitivity) in each basin (left-hand subplot) - averaged over prediction time step and lag. The alpha value (opacity) of each dot on this map is a relative measure of the fraction of the total integrated gradients of all three precipitation products (summed over time, lag, and product) due to the highest-contributing product. The right-hand subplot shows that the total integrated gradient summed over all three precipitation products is highly correlated with total precipitation in the basin.

\section{Code availability}

The code to reproduce all results and figures will be made available at https://github.com/kratzert/multiple_forcing

\section{Data availability}

The validation periods of all benchmark models used in this study are available at https://doi.org/10.4211/hs.474ecc37e7db45baa425cdb4fc1b61e1. The extended Maurer forcings, including daily minimum and maximum temperature, are available at https://doi.org/10.4211/hs.17c896843cf940339c3c3496d0c1c077. The extended

285 NLDAS forcings, including daily minimum and maximum temperature, are available at https://www.hydroshare.org/resource/ 0a68bfd7ddf642a8be9041d60f40868c/. 

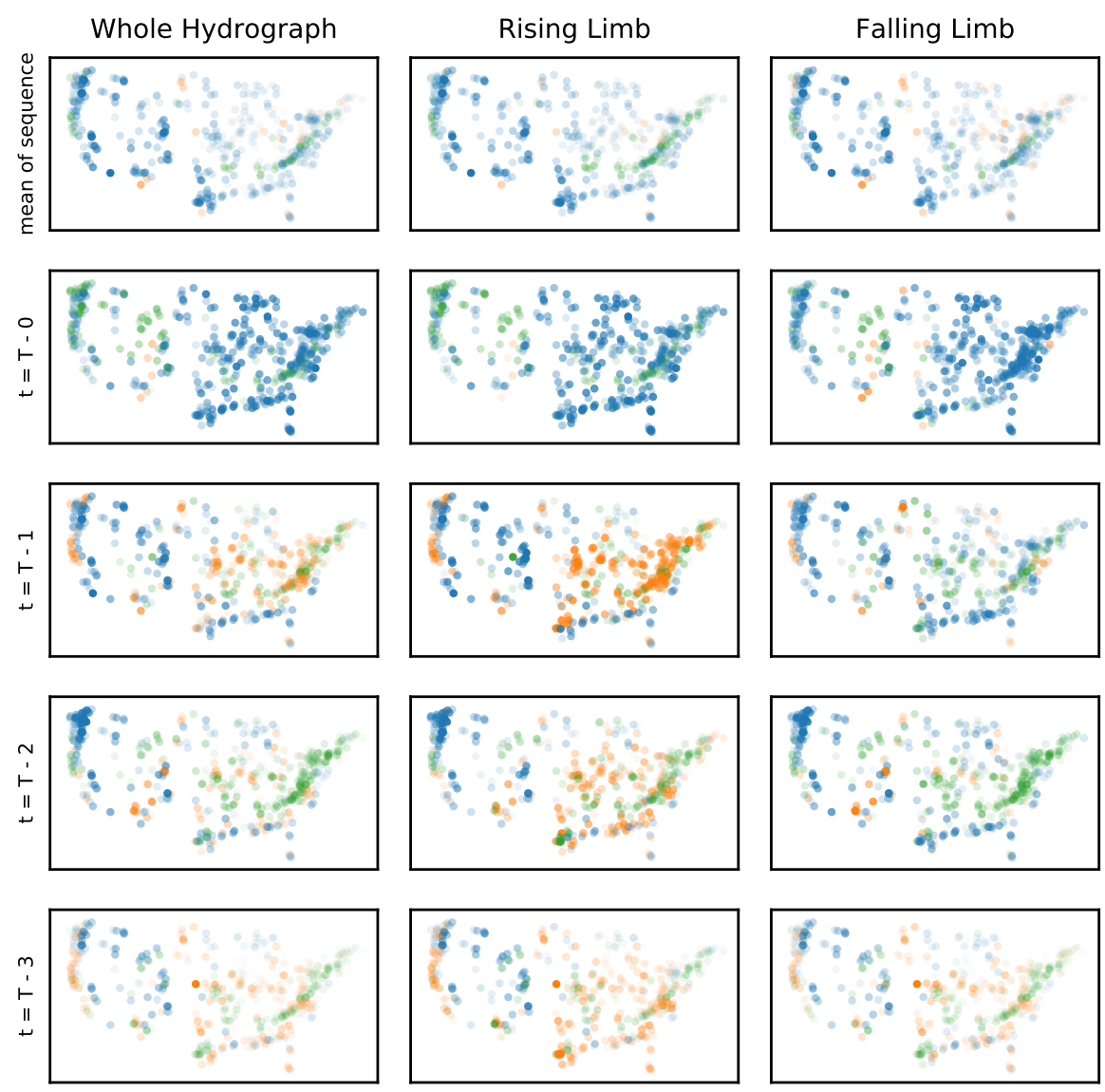

Figure 14. Spatial distribution of highest-ranked precipitation products at specific lags (different rows) over the whole hydrograph (lefthand column), and the rising- and falling-limbs of the hydrograph (center and right-hand columns, respectively), where blue circles denote DayMet, orange circles denote Maurer and green circles denote NLDAS. The take-away from this figure is that the multi-forcing LSTM learns to combine the different products in different ways for different memory timescales in different basins and under different hydrological conditions. The alpha value (opacity) of each dot is a relative measure of the fraction of the total integrated gradients of all three precipitation products due to the highest-contributing product. 
Table A1. Average single LSTM performance over a variety of metrics. The average single model performances is computed as the mean of the metric of the the $n=10$ model repetitions.

\begin{tabular}{|c|c|c|c|c|c|c|c|}
\hline & NLDAS & Maurer & DayMet & $\begin{array}{c}\text { Maurer + } \\
\text { DayMet }\end{array}$ & $\begin{array}{c}\text { NLDAS + } \\
\text { Maurer }\end{array}$ & $\begin{array}{c}\text { NLDAS + } \\
\text { DayMet }\end{array}$ & All combined \\
\hline \multirow[t]{2}{*}{$\mathrm{NSE}^{i}$ (median) } & 0.72 & 0.73 & 0.74 & 0.77 & 0.77 & 0.79 & 0.80 \\
\hline & \pm 0.003 & \pm 0.003 & \pm 0.002 & \pm 0.003 & \pm 0.004 & \pm 0.002 & \pm 0.001 \\
\hline \multirow[t]{2}{*}{$\mathrm{NSE}^{i}$ (mean) } & 0.68 & 0.70 & 0.70 & 0.73 & 0.74 & 0.75 & 0.76 \\
\hline & \pm 0.003 & \pm 0.006 & \pm 0.002 & \pm 0.003 & \pm 0.002 & \pm 0.002 & \pm 0.002 \\
\hline \multirow[t]{2}{*}{$\mathrm{KGE}^{i i}$ (median) } & 0.74 & 0.76 & 0.76 & 0.79 & 0.78 & 0.79 & 0.80 \\
\hline & \pm 0.006 & \pm 0.005 & \pm 0.003 & \pm 0.005 & \pm 0.008 & \pm 0.005 & \pm 0.004 \\
\hline \multirow[t]{2}{*}{ Pearson $r^{i i i}$ (median) } & 0.86 & 0.87 & 0.88 & 0.89 & 0.89 & 0.90 & 0.90 \\
\hline & \pm 0.002 & \pm 0.002 & \pm 0.002 & \pm 0.001 & \pm 0.001 & \pm 0.001 & \pm 0.001 \\
\hline \multirow[t]{2}{*}{$\alpha-\mathrm{NSE}^{v i}($ median$)$} & 0.83 & 0.86 & 0.86 & 0.88 & 0.85 & 0.87 & 0.88 \\
\hline & \pm 0.010 & \pm 0.011 & \pm 0.008 & \pm 0.007 & \pm 0.007 & \pm 0.005 & \pm 0.008 \\
\hline \multirow[t]{2}{*}{$\beta-\mathrm{NSE}^{v}$ (median) } & -0.03 & -0.03 & -0.03 & -0.03 & -0.03 & -0.03 & -0.02 \\
\hline & \pm 0.005 & \pm 0.004 & \pm 0.004 & \pm 0.004 & \pm 0.004 & \pm 0.002 & \pm 0.004 \\
\hline \multirow[t]{2}{*}{$\mathrm{FHV}^{v i}$ (median) } & -17.28 & -13.89 & -15.00 & -12.52 & -14.20 & -13.15 & -11.91 \\
\hline & \pm 0.904 & \pm 1.217 & \pm 0.504 & \pm 0.791 & \pm 0.881 & \pm 0.450 & \pm 0.549 \\
\hline \multirow[t]{2}{*}{$\mathrm{FLV}^{v i i}$ (median) } & -0.88 & 2.83 & 0.05 & -4.02 & 0.86 & -1.54 & 2.57 \\
\hline & \pm 7.637 & \pm 5.403 & \pm 6.056 & \pm 6.825 & \pm 5.499 & \pm 6.955 & \pm 4.072 \\
\hline \multirow[t]{2}{*}{$\mathrm{FMS}^{v i i i}$ (median) } & -9.44 & -7.31 & -5.96 & -5.60 & -7.55 & -6.93 & -6.69 \\
\hline & \pm 1.293 & \pm 1.500 & \pm 1.234 & \pm 1.241 & \pm 1.358 & \pm 0.911 & \pm 1.678 \\
\hline \multirow[t]{2}{*}{ Peak-Timing $^{i x}$ (median) } & 0.46 & 0.49 & 0.46 & 0.44 & 0.42 & 0.41 & 0.41 \\
\hline & \pm 0.010 & \pm 0.009 & \pm 0.008 & \pm 0.007 & \pm 0.007 & \pm 0.009 & \pm 0.015 \\
\hline \multicolumn{8}{|c|}{ i: Nash-Sutcliffe efficiency: $(-\infty, 1]$, values closer to one are desirable. } \\
\hline \multicolumn{8}{|c|}{${ }^{i i}$ : Kling-Gupta efficiency: $(-\infty, 1]$, values closer to one are desirable. } \\
\hline \multicolumn{8}{|c|}{${ }^{i i i}$ : Pearson correlation: $[-1,1]$, values closer to one are desirable. } \\
\hline \multicolumn{8}{|c|}{${ }^{v i}: \alpha$-NSE decomposition: $(0, \infty)$, values close to one are desirable. } \\
\hline \multicolumn{8}{|c|}{${ }^{v}: \beta$-NSE decomposition: $(-\infty, \infty)$, values close to zero are desirable. } \\
\hline \multicolumn{8}{|c|}{ vi: Top $2 \%$ peak flow bias: $(-\infty, \infty)$, values close to zero are desirable. } \\
\hline \multicolumn{8}{|c|}{${ }^{v i i}: 30 \%$ low flow bias: $(-\infty, \infty)$, values close to zero are desirable. } \\
\hline 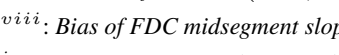 & $:(-\infty, \infty)$ & alues close t & & & & & \\
\hline
\end{tabular}


Table B1. Average single LSTM performance across a range of different hydrological signatures. The derived metric for each signature is the Pearson correlation between the signature derived from the observed discharge vs. the signature derived from the simulated discharge. The average single model performances is then reported as the mean value of the the $n=10$ model repetitions.

\begin{tabular}{|c|c|c|c|c|c|c|c|}
\hline & NLDAS & Maurer & DayMet & $\begin{array}{r}\text { Maurer + } \\
\text { DayMet }\end{array}$ & $\begin{array}{r}\text { NLDAS + } \\
\text { Maurer }\end{array}$ & $\begin{array}{r}\text { NLDAS + } \\
\text { DayMet }\end{array}$ & All combined \\
\hline \multirow[t]{2}{*}{ Baseflow index } & 0.93 & 0.92 & 0.93 & 0.94 & 0.93 & 0.93 & 0.92 \\
\hline & \pm 0.014 & \pm 0.018 & \pm 0.011 & \pm 0.005 & \pm 0.013 & \pm 0.009 & \pm 0.018 \\
\hline \multirow[t]{2}{*}{ HFD mean } & 0.95 & 0.97 & 0.97 & 0.97 & 0.97 & 0.97 & 0.97 \\
\hline & \pm 0.004 & \pm 0.003 & \pm 0.002 & \pm 0.002 & \pm 0.003 & \pm 0.003 & \pm 0.004 \\
\hline \multirow[t]{2}{*}{ High flow dur. } & 0.82 & 0.85 & 0.83 & 0.86 & 0.85 & 0.85 & 0.85 \\
\hline & \pm 0.027 & \pm 0.014 & \pm 0.010 & \pm 0.014 & \pm 0.014 & \pm 0.008 & \pm 0.014 \\
\hline \multirow[t]{2}{*}{ High flow freq. } & 0.82 & 0.82 & 0.82 & 0.82 & 0.81 & 0.81 & 0.79 \\
\hline & \pm 0.013 & \pm 0.014 & \pm 0.016 & \pm 0.016 & \pm 0.040 & \pm 0.032 & \pm 0.037 \\
\hline \multirow[t]{2}{*}{ Low flow dur. } & 0.44 & 0.42 & 0.46 & 0.47 & 0.43 & 0.46 & 0.45 \\
\hline & \pm 0.033 & \pm 0.027 & \pm 0.025 & \pm 0.035 & \pm 0.018 & \pm 0.015 & \pm 0.039 \\
\hline \multirow[t]{2}{*}{ Low flow freq. } & 0.83 & 0.82 & 0.84 & 0.86 & 0.82 & 0.84 & 0.83 \\
\hline & \pm 0.020 & \pm 0.044 & \pm 0.028 & \pm 0.022 & \pm 0.027 & \pm 0.021 & \pm 0.043 \\
\hline \multirow[t]{2}{*}{ Q5 } & 0.95 & 0.95 & 0.96 & 0.96 & 0.95 & 0.96 & 0.96 \\
\hline & \pm 0.005 & \pm 0.006 & \pm 0.003 & \pm 0.003 & \pm 0.005 & \pm 0.005 & \pm 0.003 \\
\hline \multirow[t]{2}{*}{ Q95 } & 0.99 & 0.99 & 0.98 & 0.99 & 0.99 & 0.99 & 0.99 \\
\hline & \pm 0.001 & \pm 0.001 & \pm 0.001 & \pm 0.001 & \pm 0.000 & \pm 0.001 & \pm 0.000 \\
\hline \multirow[t]{2}{*}{ Q mean } & 0.99 & 1.00 & 0.99 & 0.99 & 1.00 & 0.99 & 1.00 \\
\hline & \pm 0.001 & \pm 0.000 & \pm 0.001 & \pm 0.000 & \pm 0.000 & \pm 0.000 & \pm 0.000 \\
\hline \multirow[t]{2}{*}{ Runoff ratio } & 0.98 & 0.98 & 0.98 & 0.98 & 0.98 & 0.98 & 0.99 \\
\hline & \pm 0.002 & \pm 0.001 & \pm 0.001 & \pm 0.001 & \pm 0.001 & \pm 0.001 & \pm 0.001 \\
\hline \multirow[t]{2}{*}{ Slope FDC } & 0.62 & 0.63 & 0.59 & 0.56 & 0.59 & 0.59 & 0.57 \\
\hline & \pm 0.095 & \pm 0.053 & \pm 0.093 & \pm 0.053 & \pm 0.061 & \pm 0.091 & \pm 0.096 \\
\hline \multirow[t]{2}{*}{ Stream elasticity } & 0.61 & 0.69 & 0.70 & 0.70 & 0.68 & 0.69 & 0.71 \\
\hline & \pm 0.015 & \pm 0.024 & \pm 0.017 & \pm 0.018 & \pm 0.025 & \pm 0.032 & \pm 0.021 \\
\hline \multirow[t]{2}{*}{ Zero flow freq. } & 0.30 & 0.42 & 0.27 & 0.33 & 0.33 & 0.31 & 0.28 \\
\hline & \pm 0.101 & \pm 0.097 & \pm 0.088 & \pm 0.080 & \pm 0.067 & \pm 0.086 & \pm 0.085 \\
\hline
\end{tabular}




\section{Appendix C: Peak flow timing}

To evaluate the model performance on the peak timing we used the following procedure: First, we determined peaks in the observed runoff time series by locality search. That is, potential peaks are defined as local maxima. To reduce the number of peaks and filter out noise, the next step was an iterative process where, by pairwise comparison, only the maximum peak is kept until all peaks have at least a distance of 100 time steps to each other. The procedure is implemented in SciPy's find_peak function (Virtanen et al., 2020) and is used in the current work.

Second, we iterated over all peaks and searched for the corresponding peak in the simulated discharge time series. The simulated peak is defined as the highest discharge value inside of a window of \pm 3 days around the observed peak. And, the peak timing error is the offset between the observed peak and the simulated peak. The resulting metric is the average offset over all peaks.

Author contributions. FK had the idea for the training LSTMs on multiple forcing products. FK, DK, and GN designed all the experiments. FK trained the models and evaluated the results. GN did the triple collocation analysis, as well as the integrated gradients analysis. GN supervised the manuscript from the hydrological perspective and SH from the machine-learning perspective. GN and SH share the responsibility for the last authorship in the respective fields. All the authors worked on the manuscript.

Competing interests. The authors declare that they have no conflict of interest.

Acknowledgements. Authors from the Johannes Kepler University acknowledge support by Bosch, ZF, Google (Faculty Research Award), the NVIDIA Corporation with the GPU donations, LIT (grant no. LIT-2017-3-YOU-003) and FWF (grant no. P 28660-N31). Grey Nearing acknowledges support from the NASA Advanced Information Systems Technology program (award ID 80NSSC17K0541).

The project relies heavily on open source software. All programming was done in Python version 3.7 (van Rossum, 1995) and associated libraries including: Numpy (Van Der Walt et al., 2011), Pandas (McKinney, 2010), PyTorch (Paszke et al., 2017), SciPy (Virtanen et al., 2020), Matplotlib (Hunter, 2007) and xarray (Hoyer and Hamman, 2017) 


\section{References}

Addor, N., Newman, A. J., Mizukami, N., and Clark, M. P.: The CAMELS data set: Catchment attributes and meteorology for large-sample studies, Hydrology and Earth System Sciences, 21, 5293-5313, 2017a.

Addor, N., Newman, A. J., Mizukami, N., and Clark, M. P.: Catchment attributes for large-sample studies, Boulder, CO: UCAR/NCAR, https://doi.org/https://doi.org/10.5065/D6G73C3Q, 2017b.

Addor, N., Nearing, G., Prieto, C., Newman, A. J., Le Vine, N., and Clark, M. P.: A Ranking of Hydrological Signatures Based on Their Predictability in Space, Water Resources Research, 54, 8792-8812, https://doi.org/10.1029/2018WR022606, 2018.

Alemohammad, S. H., McColl, K. A., Konings, A. G., Entekhabi, D., and Stoffelen, A.: Characterization of precipitation product errors across the United States using multiplicative triple collocation, Hydrology and Earth System Sciences, 19, 3489-3503, https://doi.org/10.5194/hess-19-3489-2015, https://www.hydrol-earth-syst-sci.net/19/3489/2015/, 2015.

Anderson, E. A.: National Weather Service river forecast system: Snow accumulation and ablation model, NOAA Tech. Memo. NWS HYDRO-17, 87 pp., 1973.

Beck, H. E., Vergopolan, N., Pan, M., Levizzani, V., van Dijk, A. I. J. M., Weedon, G. P., Brocca, L., Pappenberger, F., Huffman, G. J., and Wood, E. F.: Global-scale evaluation of 22 precipitation datasets using gauge observations and hydrological modeling, Hydrology and Earth System Sciences, 21, 6201-6217, https://doi.org/10.5194/hess-21-6201-2017, https://www.hydrol-earth-syst-sci.net/21/6201/2017/, 2017.

Behnke, R., Vavrus, S., Allstadt, A., Albright, T., Thogmartin, W. E., and Radeloff, V. C.: Evaluation of downscaled, gridded climate data for the conterminous United States, Ecological applications, 26, 1338-1351, 2016.

Burnash, R.: The NWS river forecast system-catchment modeling, Computer models of watershed hydrology, 188, 311-366, 1995.

Burnash, R. J., Ferral, R. L., and McGuire, R. A.: A generalized streamflow simulation system, conceptual modeling for digital computers, Joint Federal and State River Forecast Center,U.S. National Weather Service, and California Departmentof Water Resources Tech. Rep., 204 pp., 1973.

Clark, M. P., Slater, A. G., Rupp, D. E., Woods, R. A., Vrugt, J. A., Gupta, H. V., Wagener, T., and Hay, L. E.: Framework for Understanding Structural Errors (FUSE): A modular framework to diagnose differences between hydrological models, Water Resour. Res., 44, 2008.

Clark, M. P., Wilby, R. L., Gutmann, E. D., Vano, J. A., Gangopadhyay, S., Wood, A. W., Fowler, H. J., Prudhomme, C., Arnold, J. R., and Brekke, L. D.: Characterizing uncertainty of the hydrologic impacts of climate change, Current Climate Change Reports, 2, 55-64, 2016.

Clausen, B. and Biggs, B.: Flow variables for ecological studies in temperate streams: groupings based on covariance, Journal of Hydrology, 237, 184-197, https://doi.org/10.1016/S0022-1694(00)00306-1, 2000.

Court, A.: Measures of streamflow timing, Journal of Geophysical Research, 67, 4335-4339, https://doi.org/10.1029/JZ067i011p04335, 1962.

Gers, F. A., Schmidhuber, J., and Cummins, F.: Learning to forget: Continual prediction with LSTM, 1999.

Gupta, H. V., Kling, H., Yilmaz, K. K., and Martinez, G. F.: Decomposition of the mean squared error and NSE performance criteria: Implications for improving hydrological modelling, Journal of hydrology, 377, 80-91, 2009.

Henn, B., Clark, M. P., Kavetski, D., and Lundquist, J. D.: Estimating mountain basin-mean precipitation fromstreamflow using Bayesian inference, Water Resour. Res., 51, 2008.

Henn, B., Newman, A. J., Livneh, B., Daly, C., and Lundquist, J. D.: An assessment of differences in gridded precipitation datasets in complex terrain, Journal of hydrology, 556, 1205-1219, 2018. 
Hochreiter, S. and Schmidhuber, J.: Flat minima, Neural Computation, 9, 1-42, 1997a.

Hochreiter, S. and Schmidhuber, J.: Long short-term memory, Neural computation, 9, 1735-1780, $1997 \mathrm{~b}$.

Hoyer, S. and Hamman, J.: xarray: N-D labeled arrays and datasets in Python, Journal of Open Research Software, 5, https://doi.org/10.5334/jors.148, http://doi.org/10.5334/jors.148, 2017.

Hunter, J. D.: Matplotlib: A 2D graphics environment, Computing In Science \& Engineering, 9, 90-95, 2007.

Kingma, D. P. and Ba, J.: Adam: A method for stochastic optimization, arXiv preprint arXiv:1412.6980, 2014.

Kratzert, F., Klotz, D., Brenner, C., Schulz, K., and Herrnegger, M.: Rainfall-runoff modelling using Long Short-Term Memory (LSTM) networks, Hydrology and Earth System Sciences, 22, 6005-6022, 2018.

Kratzert, F., Klotz, D., Herrnegger, M., Sampson, A. K., Hochreiter, S., and Nearing, G. S.: Toward Improved Predictions in Ungauged Basins: Exploiting the Power of Machine Learning, Water Resources Research, 2019a.

Kratzert, F., Klotz, D., Shalev, G., Klambauer, G., Hochreiter, S., and Nearing, G.: Towards learning universal, regional, and local hydrological behaviors via machine learning applied to large-sample datasets., Hydrology \& Earth System Sciences, 23, $2019 \mathrm{~b}$.

Kumar, R., Samaniego, L., and Attinger, S.: Implications of distributed hydrologic model parameterization on water fluxes at multiple scales and locations, Water Resources Research, 49, 360-379, 2013.

Ladson, A., Brown, R., Neal, B., and Nathan, R.: A standard approach to baseflow separation using the Lyne and Hollick filter, Australian Journal of Water Resources, 17, https://doi.org/10.7158/W12-028.2013.17.1, 2013.

Liang, X., Lettenmaier, D. P., Wood, E. F., and Burges, S. J.: A simple hydrologically based model of land surface water and energy fluxes for general circulation models, Journal of Geophysical Research: Atmospheres, 99, 14 415-14 428, 1994.

Lundquist, J., Hughes, M., Gutmann, E., and Kapnick, S.: Our skill in modeling mountain rain and snow is bypassing the skill of our observational networks, Bulletin of the American Meteorological Society, 2019.

Maurer, E. P., Wood, A., Adam, J., Lettenmaier, D. P., and Nijssen, B.: A long-term hydrologically based dataset of land surface fluxes and states for the conterminous United States, Journal of climate, 15, 3237-3251, 2002.

McColl, K. A., Vogelzang, J., Konings, A. G., Entekhabi, D., Piles, M., and Stoffelen, A.: Extended triple collocation: Estimating errors and correlation coefficients with respect to an unknown target: EXTENDED TRIPLE COLLOCATION, Geophysical Research Letters, 41, 6229-6236, https://doi.org/10.1002/2014GL061322, http://doi.wiley.com/10.1002/2014GL061322, 2014.

McKinney, W.: Data Structures for Statistical Computing in Python, Proceedings of the 9th Python in Science Conference, 1697900, 51-56, 2010.

Nash, J. E. and Sutcliffe, J. V.: River flow forecasting through conceptual models part I-A discussion of principles, Journal of hydrology, 10, 282-290, 1970.

Newman, A., Sampson, K., Clark, M., Bock, A., Viger, R., and Blodgett, D.: A large-sample watershed-scale hydrometeorological dataset for the contiguous USA, Boulder, CO: UCAR/NCAR, https://doi.org/https://dx.doi.org/10.5065/D6MW2F4D, 2014.

Newman, A. J., Mizukami, N., Clark, M. P., Wood, A. W., Nijssen, B., and Nearing, G.: Benchmarking of a physically based hydrologic model, Journal of Hydrometeorology, 18, 2215-2225, 2017.

Newman, A. J., Clark, M. P., Longman, R. J., and Giambelluca, T. W.: Methodological intercomparisons of station-based gridded meteorological products: Utility, limitations, and paths forward, Journal of Hydrometeorology, 20, 531-547, 2019.

Olden, J. D. and Poff, N. L.: Redundancy and the choice of hydrologic indices for characterizing streamflow regimes, River Research and Applications, 19, 101-121, https://doi.org/10.1002/rra.700, 2003. 
Parkes, B., Higginbottom, T. P., Hufkens, K., Ceballos, F., Kramer, B., and Foster, T.: Weather dataset choice introduces uncertainty to estimates of crop yield responses to climate variability and change, Environmental Research Letters, 14, 124 $089,2019$.

Paszke, A., Gross, S., Chintala, S., Chanan, G., Yang, E., DeVito, Z., Lin, Z., Desmaison, A., Antiga, L., and Lerer, A.: Automatic differentiation in PyTorch, 2017.

Pearl, J.: Embracing causality in default reasoning, Artificial Intelligence, 35, 259-271, 1988.

Samaniego, L., Kumar, R., and Attinger, S.: Multiscale parameter regionalization of a grid-based hydrologic model at the mesoscale, Water Resources Research, 46, 2010.

Sankarasubramanian, A., Vogel, R. M., and Limbrunner, J. F.: Climate elasticity of streamflow in the United States, Water Resources Research, 37, 1771-1781, https://doi.org/10.1029/2000WR900330, 2001.

Sawicz, K., Wagener, T., Sivapalan, M., Troch, P. A., and Carrillo, G.: Catchment classification: empirical analysis of hydrologic similarity based on catchment function in the eastern USA, Hydrology and Earth System Sciences, 15, 2895-2911, https://doi.org/10.5194/hess-152895-2011, 2011.

Scipal, K., Dorigo, W., and deJeu, R.: Triple collocation-A new tool to determine the error structure of global soil moisture products, in: 2010 IEEE International Geoscience and Remote Sensing Symposium, pp. 4426-4429, IEEE, 2010.

Seibert, J. and Vis, M. J. P.: Teaching hydrological modeling with a user-friendly catchment-runoff-model software package, Hydrology and Earth System Sciences, 16, 3315-3325, 2012.

Shrikumar, A., Greenside, P., Shcherbina, A., and Kundaje, A.: Not just a black box: Learning important features through propagating activation differences, arXiv preprint arXiv:1605.01713, 2016.

Stoffelen, A.: Toward the true near-surface wind speed: Error modeling and calibration using triple collocation, Journal of geophysical research: oceans, 103, 7755-7766, 1998.

Sundararajan, M., Taly, A., and Yan, Q.: Axiomatic attribution for deep networks, in: Proceedings of the 34th International Conference on Machine Learning-Volume 70, pp. 3319-3328, JMLR. org, 2017.

Sutton, R.: The bitter lesson, Incomplete Ideas (blog), March, 13, 2019.

Thornton, P. E., Running, S. W., White, M. A., et al.: Generating surfaces of daily meteorological variables over large regions of complex terrain, Journal of hydrology, 190, 214-251, 1997.

Timmermans, B., Wehner, M., Cooley, D., O’Brien, T., and Krishnan, H.: An evaluation of the consistency of extremes in gridded precipitation data sets, Climate dynamics, 52, 6651-6670, 2019.

410 Van Der Walt, S., Colbert, S. C., and Varoquaux, G.: The NumPy array: A structure for efficient numerical computation, Computing in Science and Engineering, 13, 22-30, 2011.

van Rossum, G.: Python tutorial, Technical Report CS-R9526, Tech. rep., Centrum voor Wiskunde en Informatica (CWI), Amsterdam, 1995. Virtanen, P., Gommers, R., Oliphant, T. E., Haberland, M., Reddy, T., Cournapeau, D., Burovski, E., Peterson, P., Weckesser, W., Bright, J., van der Walt, S. J., Brett, M., Wilson, J., Jarrod Millman, K., Mayorov, N., Nelson, A. R. J., Jones, E., Kern, R., Larson, E., Carey, C., Polat, İ., Feng, Y., Moore, E. W., Vand erPlas, J., Laxalde, D., Perktold, J., Cimrman, R., Henriksen, I., Quintero, E. A., Harris, C. R., Archibald, A. M., Ribeiro, A. H., Pedregosa, F., van Mulbregt, P., and Contributors, S. ..: SciPy 1.0: Fundamental Algorithms for Scientific Computing in Python, Nature Methods, 17, 261-272, https://doi.org/https://doi.org/10.1038/s41592-019-0686-2, 2020.

Wellman, M. P. and Henrion, M.: Explaining' explaining away', IEEE Transactions on Pattern Analysis and Machine Intelligence, 15, 287292, 1993. 
420 Westerberg, I. K. and McMillan, H. K.: Uncertainty in hydrological signatures, Hydrology and Earth System Sciences, 19, 3951-3968, https://doi.org/10.5194/hess-19-3951-2015, 2015.

Xia, Y., Mitchell, K., Ek, M., Sheffield, J., Cosgrove, B., Wood, E., Luo, L., Alonge, C., Wei, H., Meng, J., et al.: Continental-scale water and energy flux analysis and validation for the North American Land Data Assimilation System project phase 2 (NLDAS-2): 1. Intercomparison and application of model products, Journal of Geophysical Research: Atmospheres, 117, 2012.

425 Yilmaz, K. K., Hogue, T. S., Hsu, K.-L., Sorooshian, S., Gupta, H. V., and Wagener, T.: Intercomparison of rain gauge, radar, and satellitebased precipitation estimates with emphasis on hydrologic forecasting, Journal of Hydrometeorology, 6, 497-517, 2005.

Yilmaz, K. K., Gupta, H. V., and Wagener, T.: A process-based diagnostic approach to model evaluation: Application to the NWS distributed hydrologic model, Water Resources Research, 44, 2008. 